in each group gave a past history of urinary symptoms ( 37 in the 10-day group, 33 in the three-day group), and in both groups $E$ coli accounted for most of the infections (45 in the 10-day group, 42 in the three-day group).

Although reinfections were recorded as treatment failures, the cure rates obtained, nevertheless, compared favourably with figures from other series, ${ }^{8}$ in which reinfection was considered to be a failure of patient defence mechanism rather than of treatment. In our study of urinary infection in general practice the cure rate both symptomatically and bacteriologically was equally good for both treatment groups $(85 \%$ in the 10 -day group, $81 \%$ in the three-day group). We recommend the shorter, three-day course because it costs less and reduces the likelihood of patients accumulating unwanted uncontrolled drugs in their homes.
We thank Dr E A P Croydon of Beecham Pharmaceutical Division for help in designing this trial, Miss $J$ R Urwin of Bencard for undertaking statistical analysis, and Bencard for supplying Amoxil capsules.

\author{
References \\ ${ }^{1}$ Gallagher, D J A, Montgomerie, J E, and North, J D K, British Medical \\ fournal, 1965, 1, 622. \\ 2 Mond, N C, et al, Lancet, 1965, 1, 514. \\ 3 Gillespie, W A, et al, Lancet, 1971, 2, 675. \\ ${ }^{4}$ McAllister, T A, et al, Postgraduate Medical fournal, 1971, 47, suppl p 7. \\ 5 Mackey, J P, and Sandys, G H, British Medical fournal, 1965, 2, 1286. \\ 6 Charlton, C A C, et al, Postgraduate Medical fournal, 1970, 46, suppl p 30. \\ 7 Slade, N, and Crowther, S T, Britısh fournal of Urology, 1972, 44, 105. \\ ${ }^{8}$ Leigh, D A, Current Medical Research ard Opinion, 1972, 1, 10.
}

\title{
Experience of coronary care in diabetes
}

\author{
A D B HARROWER, B F CLARKE
}

British Medical fournal, 1976, 1, 126-128

\begin{abstract}
Summary
During 1968-73, 94 patients with diabetes were admitted to a coronary care unit (CCU) on 99 occasions with proved myocardial infarction. Altogether 24 of them $(25.5 \%)$ died, giving an overall mortality at the time of discharge of $24 \%$ for the total admissions. This was just significantly higher than the $19 \%$ mortality recorded among non-diabetics treated in the same period but was much lower than that among diabetics treated for myocardial infarction before the advent of CCUs. No definite correlation was found between the type of antidiabetic treatment and either mortality or the incidence of primary ventricular fibrillation. Patients with "poor" control of the diabetes before admission showed a significantly higher mortality than those with "good" control, but there was no significant difference in mortality between those with previous good control and non-diabetics.
\end{abstract}

\section{Introduction}

Reports made before the advent of coronary care units (CCU) emphasised the high mortality from acute myocardial infarction in diabetes. ${ }^{1-3}$ Apart from the results of one study, ${ }^{4}$ there is little information on mortality in diabetic patients admitted to CCUs and on whether such management has helped to reduce mortality. We have reported briefly our preliminary findings of such a study ${ }^{5}$ and present here a more detailed analysis of the results.

\section{Patients and methods}

During the six years 1968-73, 53 diabetic men (mean age $59 \pm \mathrm{SD}$ 9 years) and 41 diabetic women (mean age $61 \pm 8$ years) were admitted to the CCU at this hospital on 54 and 45 occasions respectively with acute myocardial infarction. The diagnosis was confirmed by electro-

Diabetic and Dietetic Department, Royal Infirmary, Edinburgh EH4 2XU

A D B HARROWER, MB, MRCP, senior medical registrar

B F CLARKE, MB, FRCPED, consultant physician cardiographic and enzyme changes. ${ }^{6}$ All had had diabetes for at least six months. The patients represented $2 \cdot 7^{\circ}$ o of the total admissions to the CCU for proved acute myocardial infarction over the same period, the referral area-that is, of general practices-for both diabetic and non-diabetic patients being identical. During the same period 10 further patients, all male (mean age 59 years), were diagnosed as diabetic after admission to the CCU. They are excluded from this analysis.

While in the CCU diabetic control was usually supervised by the cardiological team unless any problem arose, when a specialist was consulted. The patients' usual antidiabetic treatment was continued and modified when necessary. In accordance with the policy of the $\mathrm{CCU}^{6}$ the patients were kept in the unit for a mean of two days and then transferred to a general medical ward for further observation and management for a mean of 19 days, when they were discharged home.

Data were obtained from the records of the CCU and the diabetic department. The 94 patients (99 admissions) were divided into five subgroups according to their antidiabetic treatment, and the number on diet, sulphonylurea, biguanide, combined oral treatment, and insulin are shown in table I. Diabetic control was assessed from the mean of the blood glucose values obtained at the previous five clinic visits or during the previous two years, whichever period was the longer, with two arbitrary groups-"good" control (mean blood glucose up to $10 \mathrm{mmol} / 1(180 \mathrm{mg} / 100 \mathrm{ml})$, and "poor" control (mean blood glucose greater than $10 \mathrm{mmol} / \mathrm{l}$ ).

\section{Results}

Table I shows the mortality up to the time of discharge from hospital in the various subgroups, with respect to age and the duration of diabetes. Altogether 24 patients $\left(25^{\circ} 5^{\circ}\right)$ died, giving an overall mortality of $24^{\circ}$ for the admissions. The mortality among non-diabetics admitted to the CCU in the same period was $19^{\circ}, \ldots$ This difference is statistically significant $\left(\chi^{2}=2.85\right.$; P 0.05). The mean age at death was similar for both the diabetics (63.2 years) and non-diabetics (62.0 years). In addition, as has been found among non-diabetics treated in other CCUs, ${ }^{6}{ }^{7}$ there was a progressive rise in mortality with each decade of age (see fig).

Analysis of the relation of treatment to mortality showed that patients on diet alone had a mortality of $23^{\circ}{ }_{0}$, those given sulphonylureas, biguanides, and combined treatment had mortalities of $30^{\circ} \%$, $25^{\circ}{ }^{\circ}$, and $29^{\prime \prime}$, respectively, and the insulin group had a mortality of $17^{\circ} \mathrm{o}$. None of these differences were significant. Analysis of the individual subgroups, including all patients on oral agents, failed to confirm any significant difference in mortality from the non-diabetic patients.

Table II shows mortality related to the degree of diabetic control before admission to the CCU. In every treatment subgroup the proportion of patients with good control was greater in those who survived than in those who died, and patients with poor control had 
TABLE I-Overall mortality (CCU and wards) among diabetics with acute myocardial infarction in relation to treatment, mean age, and duration of diabetes

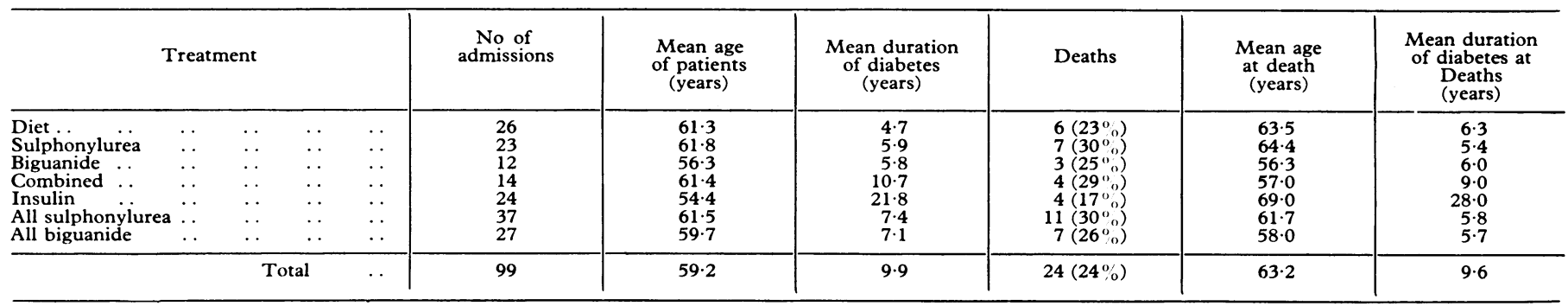

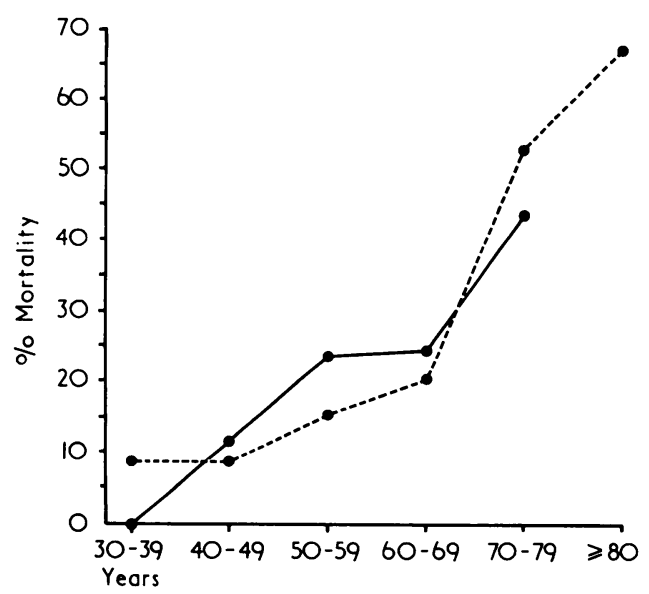

Percentage rise in mortality with age in diabetics (solid line) and non-diabetics (broken line) admitted to CCU with acute myocardial infarction.

TABLE II-Overall mortality in relation to previous control of diabetes melilius

\begin{tabular}{|c|c|c|c|c|c|}
\hline \multirow{2}{*}{\multicolumn{3}{|c|}{ Treatment }} & \multicolumn{3}{|c|}{$\%$ of patients with good control } \\
\hline & & & \multirow{2}{*}{\begin{tabular}{c|} 
Total \\
68 \\
50 \\
75 \\
29 \\
17 \\
46 \\
50 \\
\end{tabular}} & \multirow{2}{*}{$\begin{array}{c}\text { Deaths } \\
50 \\
14 \\
30 \\
0 \\
0 \\
9 \\
14\end{array}$} & \multirow{2}{*}{$\begin{array}{c}\text { Survivors } \\
75 \\
80 \\
90 \\
40 \\
20 \\
64 \\
60\end{array}$} \\
\hline $\begin{array}{ll}\text { Diet } & \ldots \\
\text { Sulphonylurea } & \ldots \\
\text { Biguanide } & \ldots \\
\text { Combined } & \ldots \\
\text { Insulin } & \ldots \\
\text { All sulphonylurea } \\
\text { All biguanide }\end{array}$ & $\begin{array}{l}\ldots \\
\because \\
\therefore \\
\cdots \\
\ldots\end{array}$ & $\begin{array}{l}\cdots \\
\cdots \\
\cdots \\
\cdots \\
\cdots \\
\cdots\end{array}$ & & & \\
\hline Total & . & $\ldots$ & 48 & 19 & 61 \\
\hline
\end{tabular}

a significantly higher mortality than those with good control $\left(\chi^{2}=\right.$ $7.66 ; P 0.01)$. In addition, there was no significant difference in mortality between diabetics with good control and non-diabetics.

Of the 24 deaths 17 occurred in the CCU and the other seven after transfer to the ward. All the deaths in the CCU were due to either progressive cardiac failure or cardiogenic shock, while five of the seven deaths after transfer were due to primary ventricular fibrillation, ${ }^{8}$ the other two being secondary to progressive cardiac failure and pulmonary embolism respectively.

Primary ventricular fibrillation occurred in 5 of the patients (three on insulin, one on sulphonylurea, and one on diet alone) in the CCU, all of whom were resuscitated. Over the same period the incidence of primary ventricular fibrillation in non-diabetics admitted to the CCU with acute myocardial infarction was $3.3 \%$. Late primary ventricular fibrillation after transfer to the ward occurred in five other patients (two on diet alone, one on sulphonylurea, one on biguanide, and one on combined treatment), all of whom died.

\section{Discussion}

The overall mortality of $24 \%$ among these diabetic patients admitted to the CCU or subsequently transferred to the general medical wards was only just significantly higher than the $19 \%$ among non-diabetics. In a similar CCU study from Birmingham, however, Soler et al ${ }^{4}$ found a much higher overall mortality among diabetics $(35.3 \%$ ) than among non-diabetics $(18 \%)$. Their patients were studied during an average hospital stay of 30 days, but further analysis of our patients for a similar period disclosed only one further death, representing an overall mortality of $25 \%$. In Birmingham $9 \%$ of all patients admitted to the CCU were diabetic compared with only $2.7 \%$ in Edinburgh. This difference is probably due to the diabetics in Birmingham being admitted from a wider area than the non-diabetics.

As the overall prevalence of diagnosed diabetes for both sexes in the Edinburgh area for patients aged $30-79$ years is $1.15 \%,{ }^{9}$ our data suggest that the incidence of acute myocardial infarction in diabetics is just over twice that expected from the prevalence of diabetes mellitus in the same population, which accords with previous evidence that diabetics are some two to three times more likely to die from coronary heart disease than nondiabetics. ${ }^{10}$ They also show that the sex ratio was $1: 1$, compared with the higher male to female ratio $(3: 1)$ in non-diabetics admitted to the same CCU. ${ }^{6}$ This agrees with previous evidence of an equal sex ratio in diabetics with myocardial infarction ${ }^{13}$ and partially reflects the high incidence of diabetes in young women with ischaemic heart disease. ${ }^{11-13}$ This again contrasts with the Birmingham study, in which a higher male to female ratio $(2: 1)$ was found in the diabetics.

The apparent, but not significant, lower mortality in the insulin-treated group may be partly explained by the lower mean age ( 54.4 years) of these patients, which accords with the rise in mortality from acute myocardial infarction with increasing age $^{6}{ }^{7}$ which was confirmed in this study. The failure to show significant differences in mortality between any of the various diabetic treatment subgroups and the non-diabetic patients may be partly attributed to the small numbers in each group, though when all the patients on oral treatment were combined, a dubious and heterogeneous group of patients with varying degrees of insulin reserve, no significant difference in mortality was found between these patients and the non-diabetics. Patients taking antidiabetic treatment by mouth, however, must have made the major contribution to the higher overall mortality in view of the similar mortality in insulin-dependent diabetics and non-diabetics. In addition, although Soler et al showed an apparently higher mortality among the patients on oral treatment than among those on insulin, their figures, like ours, were not statistically significant.

When primary ventricular fibrillation occurred in the CCU there was, as in non-diabetics, a high incidence of successful resuscitation, all five patients being defibrillated successfully with no further recurrence. In contrast, all five patients who developed primary ventricular fibrillation after transfer to the ward died. There was no difference between the early and late ventricular fibrillation groups with respect to age, previous diabetic control, history of ischaemic heart disease, or hypertension. Primary ventricular fibrillation was not a significant cause of death in diabetics in the CCU, as was also observed by Soler et al, the deaths in our series being likewise due to cardiogenic shock or progressive cardiac failure, for which coronary care can as yet contribute little, and the CCU mortality in both units was almost identical. Because of the small numbers in our series we were unable to show any increased incidence of primary ventricular fibrillation in particular subgroups. Of the 
49 patients taking treatment by mouth, however, only one developed primary ventricular fibrillation when in the CCU. As four of the five patients who developed late primary ventricular fibrillation did so within the first week after transfer and all died, there is a case for more-prolonged monitoring of diabetics with acute myocardial infarcts.

There is evidence both for $^{13}$ and against ${ }^{14}{ }^{15}$ adverse inotropic effects of sulphonylureas on the heart muscle. Further work is needed to clarify this effect, particularly in view of the evidence of increased mortality from ischaemic heart disease in patients taking antidiabetic drugs. ${ }^{16-19}$ Other factors such as diabetic control before admission may require consideration, since previous poor control appears to be an adverse factor in diabetics admitted to a CCU with acute myocardial infarction. Diabetic control in the CCU should be as good as possible, with earlier consideration for insulin treatment, as there is evidence that the ventricular fibrillation threshold is lowered in the presence of metabolic acidosis ${ }^{2}$ and raised free fatty acid levels. ${ }^{21}$

Despite our finding of an increased mortality $(24 \%)$ among diabetics admitted to a CCU as compared with among nondiabetics, this was considerably better than the $38^{\circ}{ }_{0}$ to $61 \%$ mortality ${ }^{13}$ recorded before the advent of coronary care units, and our results are not nearly so depressing as those of the Birmingham study $\left(35^{\circ} \%\right.$ ). We have also been unable to discover any definite adverse effect on the outcome of acute myocardial infarction (both primary ventricular fibrillation and mortality) from oral hypoglycaemic agents. Retrospective analyses are probably unlikely to give a clear answer to the effect of oral antidiabetic treatment on mortality in view of the relatively few patients concerned and the varying referral and admission policies of different centres.
We thank the physicians in charge of the CCU, Edinburgh Royal Infirmary, for advice and encouragement. Thanks are also due to Mrs Sadie Dickson, research assistant, and Miss Sheila M Gore, of the University of Edinburgh Medical Computing Group, for help with the statistical analyses.

Requests for reprints should be sent to Dr A D B Harrower.

\section{References}

${ }^{1}$ Bradley, R F, and Bryfogle, J W, American fournal of Medicine, 1956, 20, 207.

2 Sievers, J, Acta Medica Scandinavica, 1963, suppl No 406.

3 Partamian, J O, and Bradley, R F, New England Fournal of Medicine, $1965,275,455$.

4 Soler, N G, et al, Lancet, 1974, 1, 475.

${ }^{5}$ Harrower, A D B, and Clarke, B F, Lancet, 1974, 1, 749.

${ }^{6}$ Lawrie, D M, et al, Lancet, 1967, 2, 109.

7 Sloman, G, et al, in Symposium on Cardiac Arrhythmias, ed E Sandфe et al, p 677. Södertälje, AB Astra, 1970.

${ }^{8}$ Lawrie, D M, ct al, Lancet, 1968, 2, 523.

${ }^{9}$ Falconer, D S, et al, Annals of Human Genetics, 1971, 34, 347.

10 Pell, S, and D'Alonzo, C A, Fournal of the American Medical Association, $1970,214,1833$.

11 Bengtsson, C, Acta Medica Scandinavica, 1973, suppl No 549, p 65.

12 Mann, J L, et al, British Medical fournal, 1975, 1, 241.

13 Lasseter, K C, et al, fournal of Clinical Investigation, 1972, 51, 2429.

14 Crockett, S E, et al, Metabolism, 1974, 23, 763.

15 Young, J L, jun, et al, American Heart fournal, 1975, 89, 189.

16 University Group Diabetes Program, Diabetes, 1970, 19, suppl no 11, pp 747, 789.

17 Hadden, D R, et al, Lancet, 1972, 1, 335

18 Boyle, D, et al, Lancet, 1972, 1, 338

19 University Group Diabetes Program, Diabetes, 1975, 24, suppl no 1.

20 Gerst, P H, et al, Surgery, 1966, 59, 1050.

${ }^{21}$ Kurien, V A, and Oliver, M F, Lancet, 1970, 1, 813.

\title{
Attack rates of notified whooping cough in immunised and unimmunised children
}

\author{
NORMAN D NOAH
}

British Medical fournal, 1976, 1, 128-129

\section{Summary}

The incidence of whooping cough among immunised children under the age of six and among children of the same age who were not fully immunised was ascertained by analysing whooping-cough notifications from several health authorities. In the first three years of the study the results showed that attack rates were much higher in children who were not fully immunised than in those who were. This finding suggests that current vaccines provide young children with substantial protection against whooping cough.

\section{Introduction}

Pertussis vaccines were shown to be effective in Medical Research Council trials in $1948-52^{1-3}$ and were subsequently widely used. Later doubts about the continuing efficacy of the

Epidemiological Research Laboratory, Central Public Health Laboratory, Colindale Avenue, London NW9 5HT

NORMAN D NOAH, MB, MRCP, senior epidemiologist

vaccine $^{4-6}$ led to a Public Health Laboratory Service (PHLS) survey, ${ }^{i, *}$ which showed that much of the pertussis vaccine in general use for a few years before 1968 was not very effective. The vaccines now used differ from those assessed in the PHLS survey in being stronger (each dose containing a minimum of $4 \mathrm{IU}$ instead of $2 \cdot 1 \mathrm{IU}$ ) and in containing an adjuvant and strains of Bordetella pertussis that include the most prevalent serotype, type $1,3 .{ }^{9}$ In view of the introduction of these new vaccines it was decided to make regular comparisons of the notification rates in immunised and unimmunised children under 6 years of age. Data were provided by health authorities (see Appendix) who possessed the necessary computer facilities. These comparisons began in January 1972 and the results obtained up to the end of 1974 are reported here.

\section{Methods}

Each authority provided data on the pertussis immunisation received by each child under the age of 6 who suffered from whooping cough; the total number of children under 6 years in the area; and the number of children fully immunised against whooping cough in the area.

Children were considered to be fully immunised if they had received at least three doses of pertussis vaccine at the appropriate intervals. All other children, including those who had begun but not completed courses, were classified as not fully immunised. Most of the authorities made returns at the middle and end of each calendar year, but, owing to reorganisation, some areas were sometimes unable to supply the 